\title{
Standardized representation of typological data as common input for urban performance simulation
}

\author{
Sebastian Ebertshäuser1, Petra von Both1 \\ 1Karlsruhe Institute for Technology, Karlsruhe, Germany
}

\begin{abstract}
In the context of energetic district planning, energy simulations have steadily gained in importance through improvements and innovations in the corresponding ITsupported tools. Often, however, the continuous use of these tools up to now is hindered by insufficient data availability. Although there are technical approaches to how missing data can be supplemented across the board with the help of literature-based data that is related to the stock by the means of local typologies, these are mostly individual approaches of single experts. In order to create a uniform basis for this type of information requirements, an extension of the established object model CityGML was developed within the framework of the research project EnEff:Stadt-ModelSIM. With the concept prepared as application domain extension (ADE), typological data can be described in the urban models and make it possible to support town planning throughout, i.e. accompanying the process.
\end{abstract}

\section{Introduction}

As complexity in terms of pressure, e.g. to achieve timeframed global goals regarding climate change, diversity, e.g. of different themes or involved stakeholder, duration, e.g. of planning phases as well as corresponding planning horizons, is continuously on the rise, an integrated planning of urban (energy) systems has to be increasingly supported by IT-based planning and simulation tools. Up to now various existing simulation tools share a common problem as their capabilities rely on the data that is available for the local situation that shall be analyzed. Here, there often is a lack of comprehensive and up-todate information on the build environment and energyrelated infrastructure as needed for input to the energetic simulation. On side of assessment methods therefore there is a need for typology data that can help to fill the information gaps in specific data available for the local context. However, again due to missing common data the compilation of these typological information on base of literature data is often individually done by experts in a locally specific manner that can't be generalized. This contribution presents findings that address this problem field from the ongoing EnEff:Stadt-ModelSIM research project that pursues solutions for energetic urban simulation that base to the most extend on established standards (see Figure 1 that depicts the approach of the project).
For the side of simulation technology on the level of building there has been vast developments in standardized and therefore interchangeable performance simulation technology within the framework of Modelica (IEA Annex 60, 2017). This object-oriented simulation standard that was developed in the field of automotive production enables for a component based structuring of the simulation models (Modelica Association, 2019). Thus, in terms of building performance simulation (BPS) different Modelica libraries have been developed and efforts undertaken to base them upon a common foundation (IEA Annex 60 Modelica, 2017) in order to build simulation models for a building that is put together from the various component models in the different libraries. As this effort has been accomplished to some extend and is currently further optimized (IBPSA Project $1,2019)$, the next frontier for the content of the developed libraries is to be combine into different building simulation models in order to accomplish simulation on the urban scale. For this side of simulation within the framework of the research project underlying this contribution a partner is involved that develops one of these Modelica component libraries for BPS.

On side of urban data, a common language for exchanging the various information involved in the urban planning processes can be found in the well-established objectoriented OGC standard CityGML (OGC, 2012). The standardization initiative SIG3D (2019) is the driving force behind the development of this international OGCnormed standard. Therefore, for data integration as base of generalized simulation approaches it provides a robust open standard within the heterogeneous landscape of GIS databases implemented by communities. Also driven by the success of the top-down initiative of the European Union to standardize (urban) data, INSPIRE (European Commission, 2019) as well as its national implementation initiative GDI-DE (2018) (from within which SIG3D was founded), many German communities already have built up databases that are able to import/export the standard or natively base upon it. As a common source of 3Dinformation on the built environment the model standard is predestined for urban (energetic) performance simulation. Thus, it can help to streamline efforts for data acquisition and comparison of respective results. At core of the CityGML-schema stands the Level-Of-Detail (LOD) concept to model (semantic and geometric) information in different granularities. This can facilitate in principle the depiction of different kind of information 


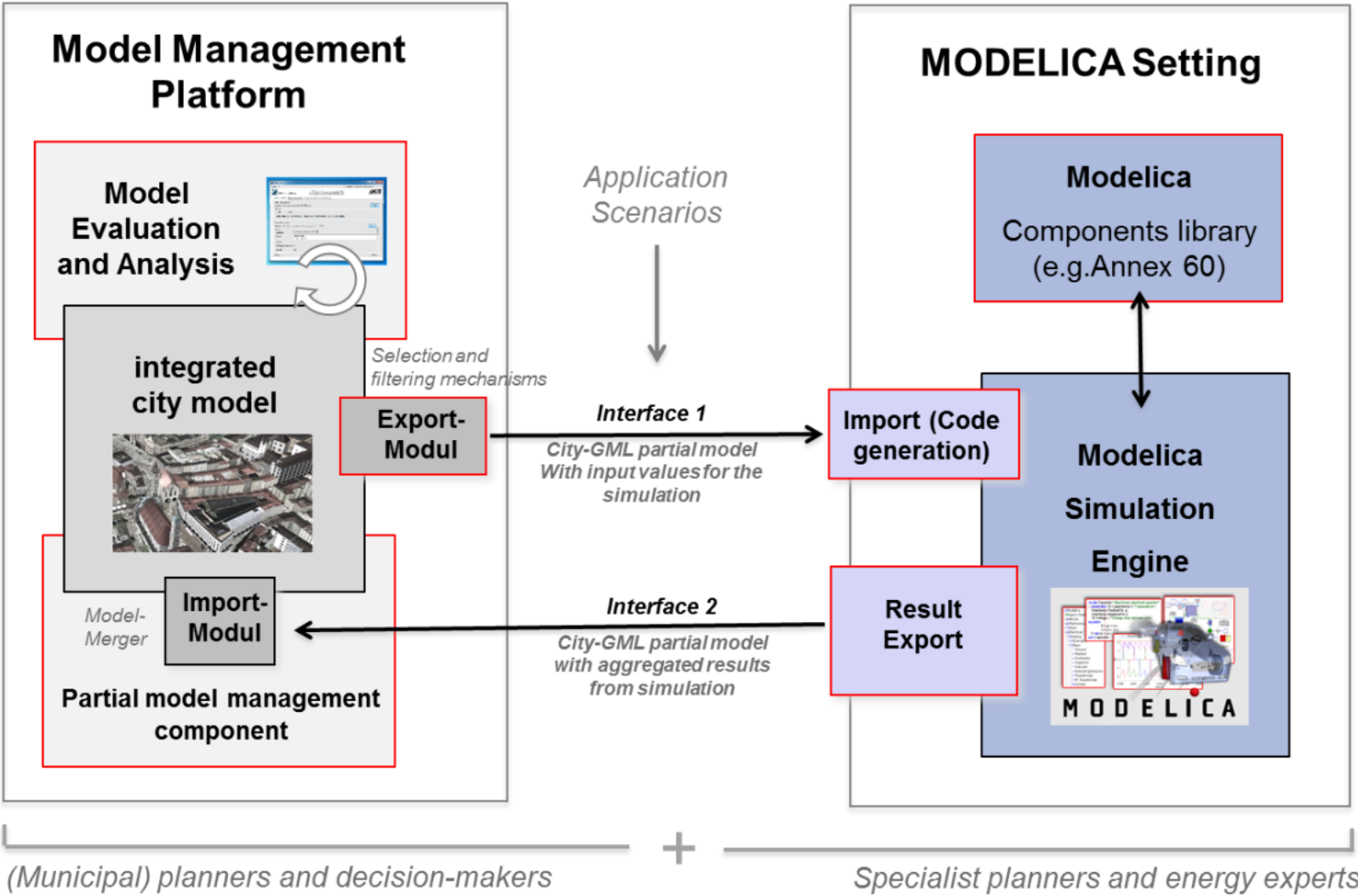

Figure 1: Target process of the collaborative project.

granularities integrated in one urban model as often found real-life situation of data availability in the communities.

But despite of a continuous enhancement of the schema, the standard still lacks depiction concepts for literaturebased input data (e.g. typological building information), that allows filling the gap of often missing local buildingspecific data as indicated above for energetic urban simulation. With the Application Development Extensions (ADE) the standard CityGML at its core provides a mechanism for extension that is also used officially by the standardization organization to preliminary extend the model semantics in the current version as preparation of the next release. In recent years due to the utilitarian use of CityGML developments of $\mathrm{ADE}$ are got more and more popular to cover in a standard way various fields of information demands with specifically needed semantics. For instance, the ENERGY ADE is being officially developed by SIG3D for the broad thematic field of urban energetic information (SIG3D, 2019). In this ADE development, however, the depiction of typological data has not yet been considered, e.g. information regarding building typology with further properties representing the single archetypes that are then mapped to the concerned buildings in the urban model. Since in urban performance simulation this information plays an important role to approximate data, that is not available for a local situation, a standardized way of describing this input data would enhance the reuse possibilities of simulated results. Especially as it would be clearly indicated on which kind of input data they base upon.

\section{Methods}

From the findings of the underlying research project EnEff:Stadt-ModelSIM this contribution presents a suggestion for extending the CityGML standard towards typological data within the framework of an ADE development, aiming at better comparison of simulation results by enabling consistent references to - either real world or typological mapped -inputted data.

Although there is no norm that describes the generation of an ADE explicitly, various developments were examined (e.g. Brink, 2013) and a common methodology is adapted on how to best extend the structures of CityGML. In contrast to the building level, where the norm-based procedure of the Information Delivery Manual (IDM) with the accompanying Model View Definition (MVD) Standard is available for the specification of own standards-based information requirements on the basis of the established open building information model standard IFC, the CityGML ADE mechanism is based on pure XML schema language XSD only. However, the OMG Unified Modeling Language (UML) notation standard has established itself as a uniform development basis for the graphic representation of information structures in a concept model in the standards based on the OGC basic schema Geographic Markup Language (GML). However, a norm-based transfer logic from the diagrams noted in UML to corresponding XSD entities (XSD profile for the UML) is missing in case of the extending the CityGML standard. For this purpose, it was possible to build on the modeling best practices (ibid.) published within the framework of various ADE developments, as well as on 


\section{ModelSIM ADE}

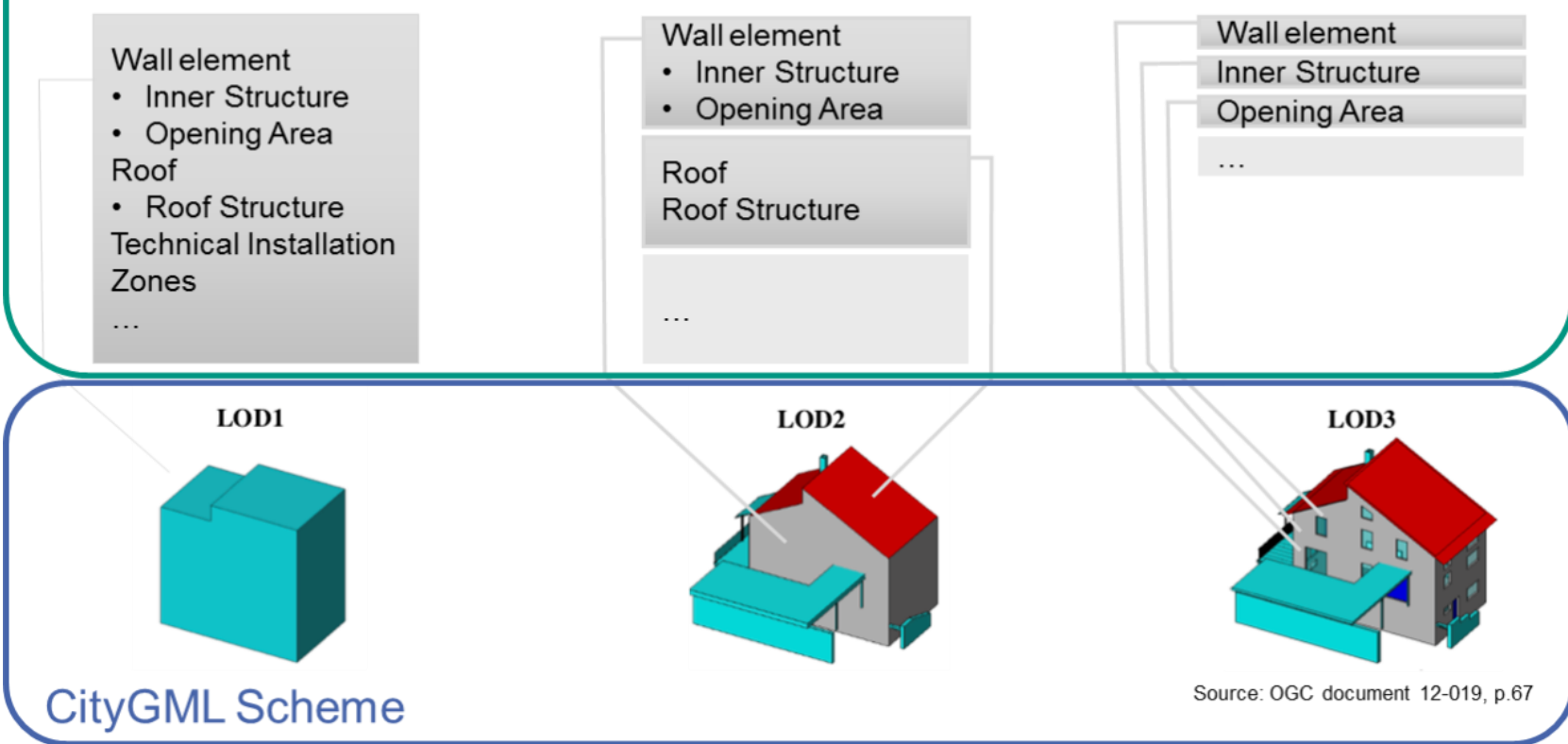

Figure 2: Concept to semantically extent the _CityObject at CityGML's core.

the experience of members of the SIG3D standard committee who are currently involved in the development of official ADEs, such as e.g. ADE Energy. This together with the involvement of respective UML to XSD transformation tools of these experts builds the (technical) generation context of the presented depiction concept for typological data. The information scope of typology data taken into account are:

a) methods for typology generation

b) thematic field regarding buildings and energy

Firstly, in order to achieve the best added value for the proposed information structure, as a base for depiction of the methodical parts the approach of the TABULA building typology (IWU, 2016) was chosen as blueprint. As a (referable) published method it stands out of the usual practice that is dominated by individually tailored and thus difficult to normalize approaches.

Secondly, for proposing a realistic interface to exchange data in urban planning, a consistent description of an appropriate application context is crucial. The proposed conceptual background is derived from a use-case assessing potential areas for district heating as defined in the EnEff:Stadt-ModelSIM project (Ebertshäuser, 2018).

The analysis of a required bidirectional data exchange between the municipal planning and the technical simulation side resulted in four strands of information requirements as a basis for the specification of an ADE for this application case "Identification of variants for district heating priority areas". With these initial data strings of the application case listed in the following, the different granularities of the local data availability to the building stock should be taken into account and the developed interface should thus cover as broad a spectrum of municipal situations as possible.

- Specific consumption-based building data (often available from local energy suppliers)
- Specific demand-oriented building data (e.g. collected within the framework of regulatory regulators)

- Typological consumption-based building data (e.g. empirical studies on consumption of different building types)

- Typological demand-oriented building data (e.g. literature-based studies on construction types calculated according to national energy balance procedure, Loga, 2012)

A two-stage description concept for the implementation of the ADE specification was developed as the basis for the depiction of these four strands.

\section{Extension concept for source object_CityObject}

With the object-oriented city model standard CityGML, (existing) parts of the city such as buildings, streets, furniture etc. but also the natural environment with water areas, vegetation etc. can be described. In order for these depiction concepts to be extended for the application context of a connection to Modelica-driven city simulation, respective possibilities to describe the needed energy-related information must first be added on the semantics side. The aim is to descript the native CityGML entities in their inner constitutions as they are originally designed to represent the visible surfaces of things (in the city) alone.

In order to take into account, the topology of the standard, i.e., for example, the part-whole relationship of a building to its walls as well as their relationship to their respective openings, the extension should be based on CityGML's abstract source object_CityObject. In principle it is thus possible to describe the "contents" of the building object bldg:Building, as well as those of all native CityGML entities (roads, bridges, etc.) or those of other elements added in an ADE uniformly. It is also important to bring the existing geometric LOD detailing concept into the 
extension approach. Figure 2 schematically illustrates this conceptual extension on behalf of the Building object. If, for example, only the building object and no or only the building outline is available as geometry from the native CityGML side (cf. LOD 0 or 1), a bundled data set is appended to the object. If detailed (geometrical) descriptions of the structure are available, such as the individual wall and roof surfaces in LOD 2, the conceived extension concept sees the decomposition of the dataset of the building object into the component objects wall and roof. The geometric characteristics described alphanumerically in the summarized case alone, such as surface or orientation, are implicitly assigned to the natively existing geometric descriptions of the component objects in LOD 2. Furthermore, in contrast to the "thermal" surfaces mapped redundantly to the native geometry in Energy ADE, the (re-)use of the native geometry description within the framework of the extension concept, e.g. window or opening area (cf. Figure 2, right-hand side), keeps the size of the corresponding model instances considerably smaller in terms of data technology.

\section{Depiction concept for typology data}

The concept for depiction of typological data is based on above described extension to CityGML's source object for the supplementary description of energy-relevant "contents" that applies to all native entities of the model standard (see Figure 3). The basic (methodological) components used by TABULA for a building typology are taken up and converted into a corresponding part of this depiction concept. The principle pursued here starts with the possibility of being able to introduce a typology or taxonomy object into the city model as an information reference point for the respective typology data. However, so that not all (static) information concerning the typology has to be kept in the model instance, the concept is strongly based on data stored externally in catalogs that is referenced from the entities included in the model (see Data Dictionary in chapter Results). The now implemented basic components of the TABULA typology method are on the one hand the different classification systematics, like age classes, function, form etc., as well as on the other hand the further characteristics to their thereby differentiable archetypes. The description concept therefore provides for the addition of an archetype object derived from the extended building object (see above) for each archetype of the typology. This has properties that can be assigned using references to corresponding catalogues with classification characteristics. For example, an appropriate archetype can then be created for an apartment building (MFH) by reference to an entry in the "function catalogue" together with a reference to the construction age class 2010-2015. This can then be further specified via the underlying extended building model. Thus, TABULA provides socalled "sub-typology" classification features with which e.g. construction types or type of technical installation system can be further differentiated. The possibilities of description in the archetype allow this further differentiation on the one hand by referencing corresponding catalogues in the "inner" properties added to the building model, e.g. for the property "wall construction" a catalogue reference to the particular construction type etc., on the other hand by describing any other (energetic) characteristics. In empirical studies, for example, a wide variety of characteristics to archetypes e.g. of Tabula have been investigated, in part by the area on which the study is based with regional peculiarities, which can be supplemented by means of the description concept without altering the typology characteristics that may be generally underpinned. In principle, the flexible approach allows both the complete description of an archetype as a nested instance in the typology object

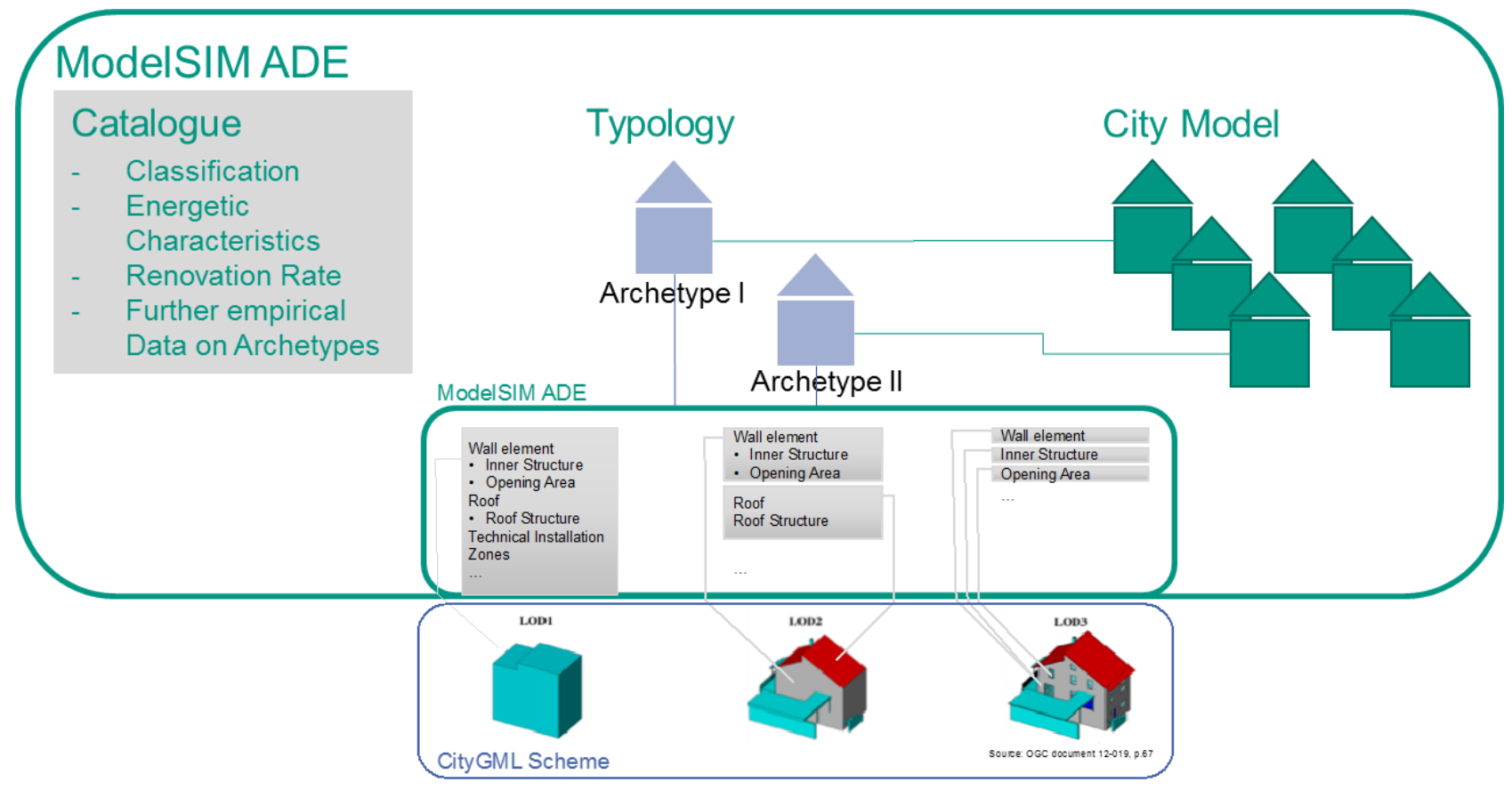

Figure 3: Concept to depict (and reference) typoloogical urban data. 
within the city model, as well as a corresponding reference to an externally held archetype object or combinations thereof. So that a building in a city model can also be assigned to different archetypes, for example from different building typologies described in one city model instance, the archetype object derives from the native CityObjectGroup base class. Because only the archetype knows its "occurrences" in the city model, but not vice versa, it is thus possible to update and refine the typological information provided in the model without changing the native CityGML data on the actual building stock.

\section{Results}

The implementation of the ADE within the framework of the EnEff:Stadt-ModelSIM project is based on the steps described above:

- Application context with process

- Clarifying information needs (Loga, 2012)

- Conception of an (extended) depiction concept in comparison with CityGML

In the following, the schema constructs of the ADE that were transferred from the depiction concept to a formal UML concept model are presented. With excerpts from XML instance documents that follow this schema, these are further illustrated.

In Figure 4, the basic extension of the CityGML source object _CityObjekt is shown in sections of the concept model. This entity is supplemented with two fields. To depict the internal material structure of the object, the field consistsOf can be assigned with an instance of the specialization class derived from the base class AbstractConstituent. In principle, the physical (component) structures described in this field can be used (according to the level of detail of their description) to assign material(s) to each CityGML object. For example, an instance of the derivation class of the base class AbstractMatter assigned to the field consists $O f$, which represents "amorphous" water in the case of native WaterSurface objects or other instances of derived classes thereof representing a single material or bundle in the case of components of a native Building object. In addition to depiction of this physical structure, the portionedBy field of an object can be used to describe an inherent or intended (logical) structure by assigning an instance of the specialization class of the base class LogicalStructure. In general, the extension principle follows the example of the building information model IFC with the separate description of physical or logical structure (for example, spatial structure in the special case of an extended Building object). The division therefore allows not only the flexible description of a concrete building but also in particular the definition of general construction, room or zone types. This type information can be included in any combination in the concrete description of the building instance (for example, to close information gaps in a particular building with typical information).

In addition, they can also be stored as general catalogues of common information that can be referenced in the city model (for example, they can also be assembled as an archetype in the context of a building).

The hierarchies of the specialization classes for the two abstract base classes AbstractConstituent and LogicalStructure are shown using the example of the building object in Figure 4 on the right-hand side. In a native (geometric-less) CityGML LOD 1 building, for example, the consists $O f$ field can be assigned with an instance of the ConstructionSystem class that contains all further energy-relevant information on the physical structure in alphanumeric form. These could then be the parts of the building that are pronounced as ConstructionElement objects, such as roofs, ceilings,

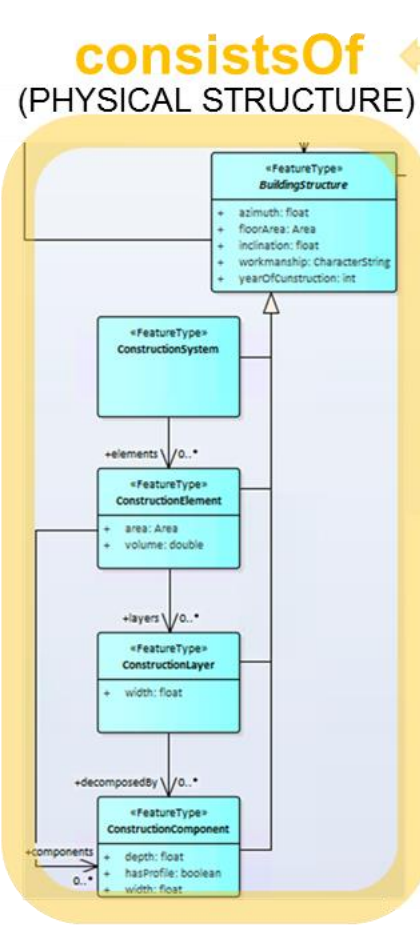

Connection to Material further decomposition of CityGML's core_CityObject Element

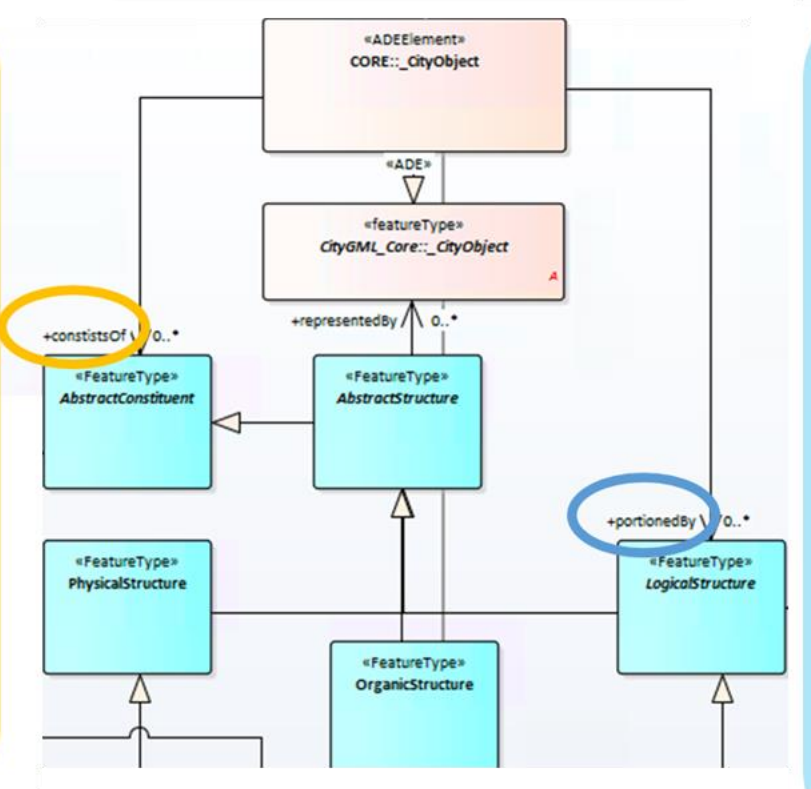

Figure 4: UML diagram of extension methodology.

\section{portionedBy} (SPATIAL STRUCTURE)

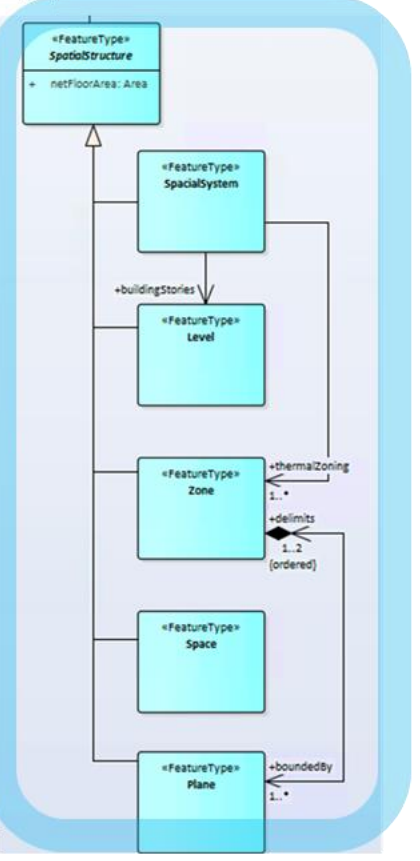


walls. Furthermore, these could also be broken down into further components and described in more detail.

For example, it is possible to emboss a wall component object in its layers field with individual ConstructionLayer objects that represent its individual wall layers. Also on the side of the spatial structure a finegranular building topology can be described by the extension already at the LOD 1 building also without detailed geometrical representation. In order to achieve this the portionedBy field of the native Building object is assigned with a SpacialSystem object, which represents the topology of the whole building. Without further subdivision, all (geometric) parameters, such as normbased surfaces or volumes, can already be described alphanumerically in this object.

For further, finer granular depiction of the topology, it is also possible to formulate detailed levels of the building in the field stories by means of assigning respective Level objects or describe the thermal zoning of the building in the field thermalZoning with different pronounced Zone objects. As shown in the concept sketch (see Figure 2), this single data set in the case of an LOD 1 model for describing the energy-relevant information that is nested in the consists Of or portionedBy fields of the building object can be further subdivided into the native CityGML model topology if a respective greater LOD is given. Figure 5 shows this using the example of a LOD 3 model. The consistsOf field of the superordinate native component WallSurface is assigned with a ConstructionElement object that carries the energetic properties of the wall. In this wall object, the wall layers are also nested as ConstructionLayer objects in the layers field. Thus, the layers share with the wall object the

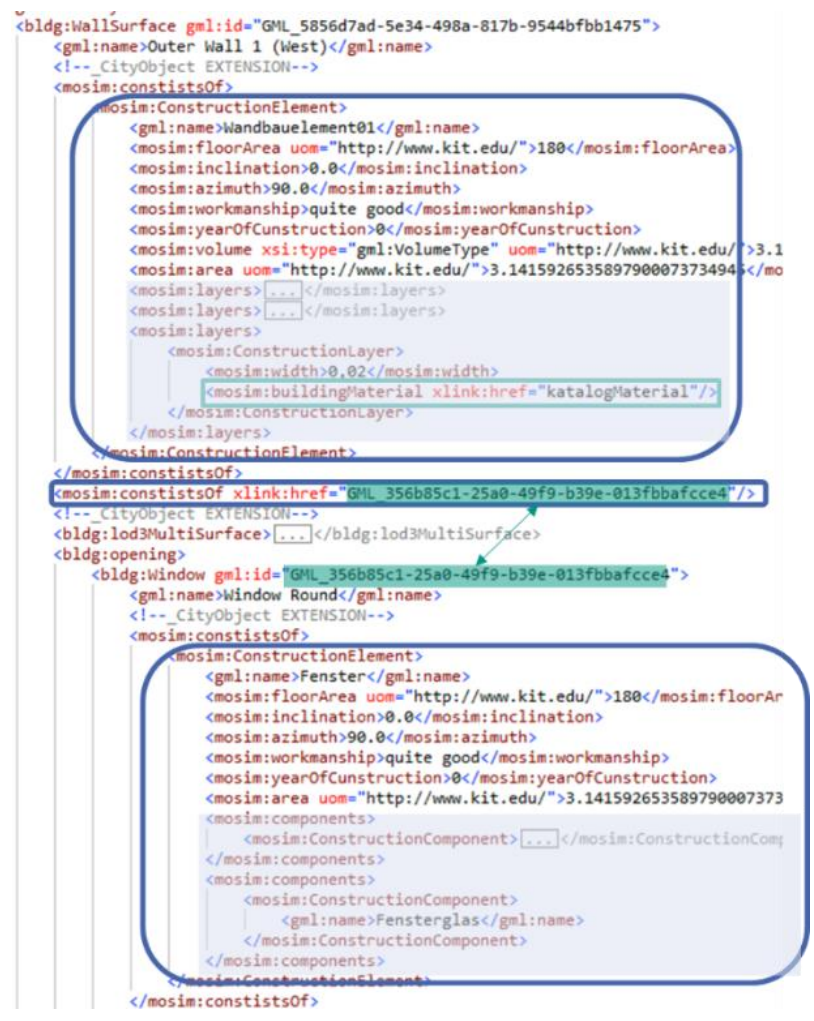

Figure 5: Example Building excerpt in XML instance document. natively geometrically (GML-based) described area, since they are all contained in the WallSurface object for the LOD (and need therefore no more own alphanumeric area specification). Instead of assigning a ConstructionElement object representing the window, as in the case of LOD 2 and smaller, in the component field of the wall object, it can be located in the Window object residing natively as the opening surface in the LOD 3 WallSurface object. For this, the corresponding field consists Of of the Window object can be assigned with the ConstructionElement object, which represents the energetic properties of the window, and is thus implicitly coupled with the window area also geometrically described in the Window object. In addition, ConstructionComponent objects representing the parts of the window can be added in the components field in order to describe the window frame or the glass more precisely.

\section{Depiction of Typology Data in the Concept Model}

The extension concept of the source object _CityObject shown in Figure 4 as UML concept model as well as the corresponding excerpts of a XML instance document (Figure 5) forms, as already schematically shown in Figure 3, also the basis for the mapping concept of the typological data. In the root node of the CityGML instance document, the CityModel object, a corresponding Typology object is first created in the typologyMembers field to instantiate a typology. The further concrete design of this object depends strongly on whether the information contained is held in the model itself or whether it is referenced via reference elements in external catalogs. When designing the structure of the external data dictionaries in the case of catalogue data, the SimpleDictionary class, which is also defined by the native CityGML via its basic schema GML, was predominantly used. Figure 7 shows both principally possible characteristics of the instantiated Typology class in the excerpt of a XML instance document. Thus, on the one hand side archetypes of the typology can be referenced as complete data records by the field externalReference in an external data dictionary. In the items field, an ArcheType object is created for each of the archetypes (Figure 6). This object on the other hand side can also be used to create complete information on the archetypes in the city model instead of externally referenced data sets. As a derivative of the CityObjectGroup class, the instantiated ArcheType object inherits the mechanism for grouping objects to reference occurences of this archetype in the city model instance.

In the ArcheType object, the archetype field is then assigned with a corresponding representative of the basic type of typology, in the example shown in Figure 8 a specialized building ArchetypeBuilding.

For the depiction of a building typology, the native Building object already supplemented by the extension of the source object_CityObject was derived and supplemented by properties specifically required for the description of an archetypal building. Thus, for example, the classification features, e.g. construction age class, were added. 


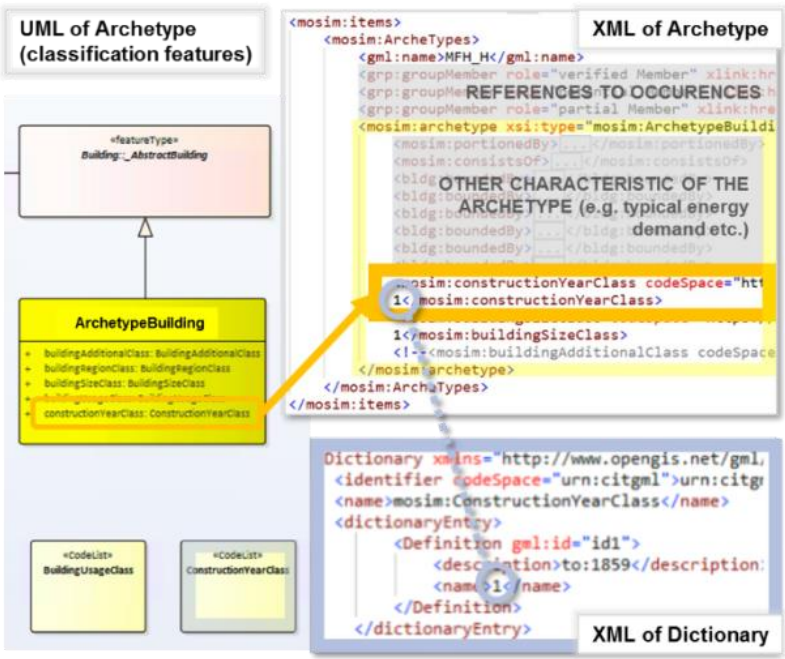

Figure 6 Connection of typological classification features in external catalogues

If the classification characteristics are assigned with corresponding references to the classification catalogs, the Archetype object can be further pronounced by its fields consistsOf or portionedBy inherited from the extended building object and already introduced above. For example, the other sub-classification characteristics can be described as exemplified in Figure 8.

Instead of nesting into a single ConstructionSystem object in the field consists Of of ArchetypeBuilding detailing the energetic properties, the example is based on a LOD 3 model. As already indicated above with the extension of a concrete building, typical constructions, like e.g. the envelope components or the roof, can be assigned as respectively detailed ConstructionElement objects in the native CityGML description of the parts of a house, which are created as WallSurface or RoofSurface.

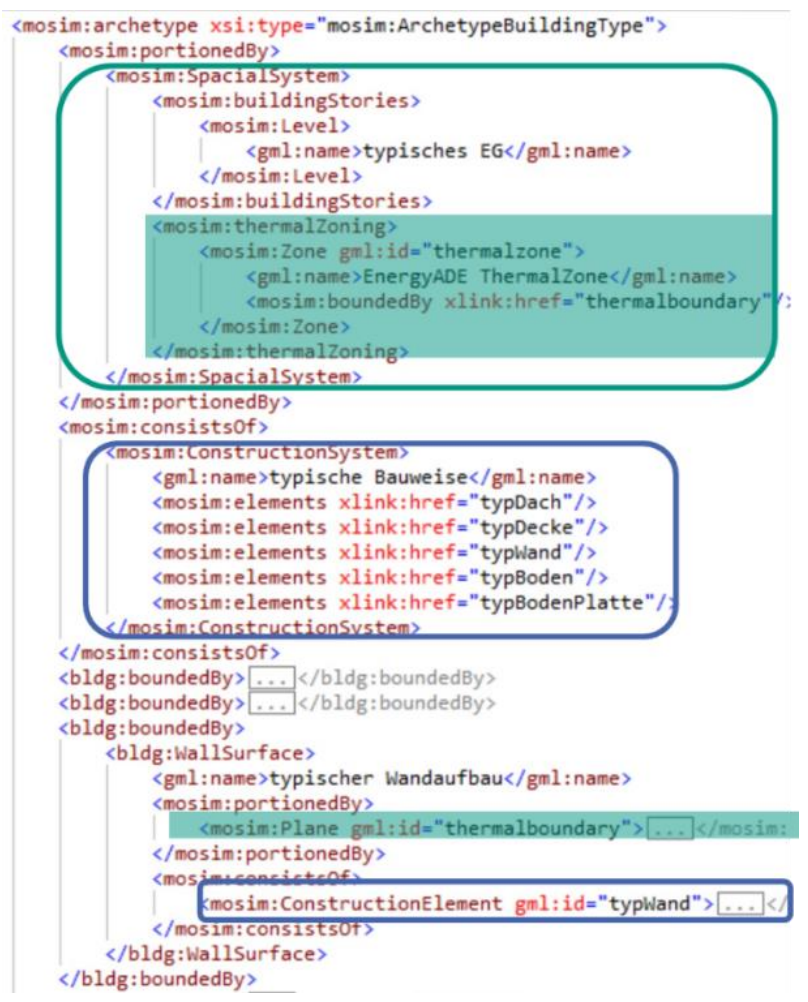

Figure 8: Example ArcheType excerpt in XML instance document

\section{Conclusion}

On behalf of two fundamental depiction conceptions at the core of an ADE development one part of the ongoing

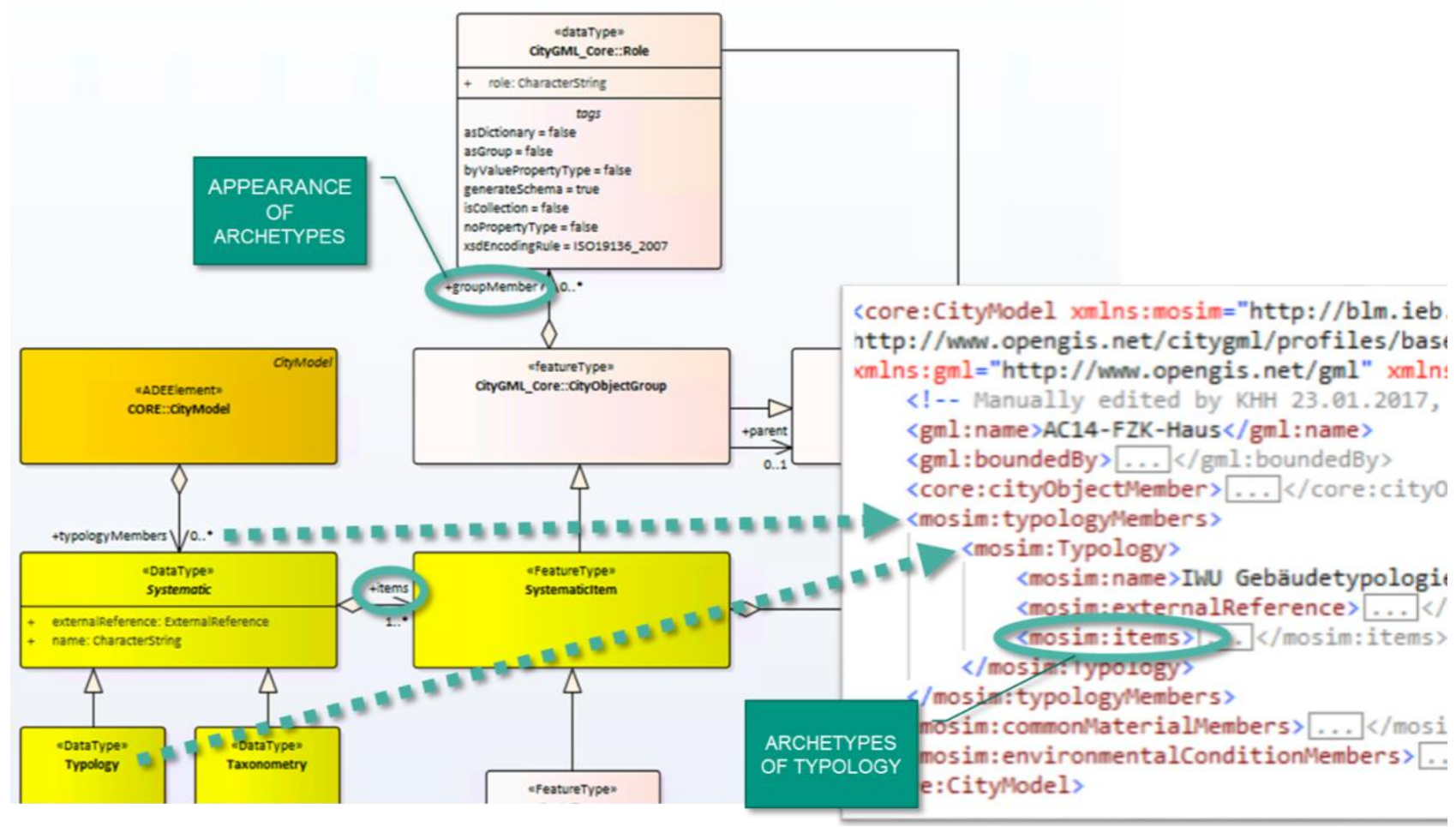

Figure 7: Excerpt of concept model for typology data and e xample XML-instance document Typology object. 
EnEff:Stadt-ModelSIM project could be illustrated above. The aim of extending the established OpenGIS standard CityGML thereby lies in enabling seamless connection of the urban data that can be carried within its structures to the side of simulation with the open Modelica language. Thereby, comprehensively analyzing possibilities to adapt structures on both sides for a better mapping of the concrete information on the building stock that can then be used for parametrizing component models that have been developed in the recent years within different Modelica libraries for BPS. The developed interface for which currently within the framework of the project a respective toolchain is being developed is capable of returning the results of simulation back to the side of town planning. Thereby especially the reference on the information that was used as input to generate the results enables for a transparent and useful data format for this purpose.

As the scope of the interface was limited to the use case "Identification of variants for district heating priority areas" of the research project EnEff:Stadt-ModelSIM in order to further disseminate the proposed scalable information structures that were formalized in the ADE two strategies are being pursued. On the side of standardization, a close exchange with experts as well as inputs to the respective working groups is undertaken by the project members. Here, especially the official Energy ADE development is of interest. The presented concepts are on side of modeling in the background being prepared to be selectively proposed as supplementary parts into this broader standardization effort in the field of urban energy data.

On the side of the established open simulation language Modelica the proposed depiction of typological data will be contributed into ongoing efforts to further standardize component models in the field of BPS. Here, within the framework of IBPSA the important work of harmonizing different component model libraries that was started in the international IAE Annex 60 is being continued. Within the IBPSA Project 1 therefore, besides the optimization of the component libraries themselves the use of the models for urban energy simulation is particularly addressed. In this context the proposed ADE as CityGML extension of the EnEff:Stadt-ModelSIM project is one of the deliverable for the corresponding work package that streamlines the efforts in terms of Modelica and simulation on urban scale.

\section{Acknowledgement}

The research project EnEff:Stadt-ModelSIM on which this contribution is based was funded by the German
Federal Ministry for Economy and Energy (BMWi), funding code 03ET1410.

\section{References}

Brink, Linda van de et.al (2013); UML-Based Approach to Developing a CityGML Application Domain Extension. In: Transactions in GIS; Volume 17, Issue 6, wiley. https://doi.org/10.1111/tgis.12026

Ebertshäuser, S. et. al. (2018): Unterstützung kommunalplanerischer Prozesse mit CityGMLbasierter Anbindung Modelica-getriebener Quartierssimulationen; BauSIM 2018 - Tagungsband der 7. Deutsch-Österreichischen IBPSA Konferenz. Karlsruhe (Germany), 26-28 September 2018.

European Commission (2019) INSPIRE Knowledge Base, https://inspire.ec.europa.eu/, accessed 02.01.2019

GDI-DE (2018). Geodateninfrastruktur Deutschland website, http://www.geoportal.de/DE/GDIDE/Organisation/organisation.html, accessed 05.09.2018

IBPSA Project 1 (2019) Project 1 Repository, https://ibpsa.github.io/project1/, accessed 03.02.2019

IEA Annex 60 (2017). Website, http://iea-annex60.org/, accessed 02.11.2018

IEA Annex 60 Modelica (2017). Library with models for building energy and control systems, http://www.ieaannex60.org/releases/modelica/1.0.0/help/Annex60.h tml, accessed 11.06.2018

IWU (2016). Project Tabula Typology Concept, http://episcope.eu/building-typology/tabulastructure/concept/, accessed 10.08.2018

Loga et. al (2012); Typology Approach for Building Stock Energy Assessment. Final Project Report, InstitutWohnenundUmweltGmbH, Darmtadt Germany. http://www.buildingtypology.eu/downloads/public/docs/report/TABULA _FinalReport.pdf

Modelica Association (2019). Official Modelica Website, https://www.modelica.org, accessed 02.01.2019

OGC (2012) OpenGis Standard CityGML https://www.opengeospatial.org/standards/citygml, accessed 03.05.2018

SIG 3D (2019) Special Interest Group 3D Website, https://www.sig3d.org/index.php/de/standardisation.h tml, accessed 03.03.2019 